(1)

CrossMark

\title{
Insulin resistance modifies the association between obesity and current asthma in adults
}

\author{
Juan Carlos Cardet (1) ${ }^{1,2}$, Samuel Ash², Tope Kusa ${ }^{3}$, Carlos A. Camargo Jr ${ }^{4}$ and \\ Elliot Israel ${ }^{1,2}$
}

Affiliations: ${ }^{1}$ Division of Rheumatology, Immunology, and Allergy, Brigham and Women's Hospital, Boston, MA, USA. 'Division of Pulmonary and Critical Care Medicine, Brigham and Women's Hospital, Boston, MA, USA. ${ }^{3}$ Dept of Epidemiology, Harvard T.H. Chan School of Public Health, Boston, MA, USA. ${ }^{4}$ Dept of Emergency Medicine and Division of Rheumatology, Allergy, and Immunology, Dept of Medicine, Massachusetts General Hospital, Boston, MA, USA.

Correspondence: Elliot Israel, Brigham and Women's Hospital, 75 Francis St, Boston, MA 02115, USA.

E-mail: eisraeldpartners.org

ABSTRACT Insulin resistance potentiates the association between obesity and childhood asthma, but this relationship appears inconsistent in relatively small studies of adults. We investigated effect modification in adults using the National Health and Nutrition Examination Survey 2003-2012, a large, nationally representative database.

Insulin resistance and a history of physician-diagnosed current asthma were obtained from 12421 adults, ages 18-85 years. We used logistic regression to determine associations between obesity and current asthma, adjusting for age, sex, race/ethnicity, poverty income ratio and smoking status. An interaction term evaluated effect modification by insulin resistance of the obesity-asthma association.

As expected, obesity was positively associated with current asthma. Insulin resistance modified this association, with obesity measured as body mass index, waist circumference or waist-to-height ratio. The relationship between obesity and current asthma was stronger with increasing insulin resistance tertiles (OR 2.05, 95\% CI 2.76-3.00; p-value for interaction 0.03). This association was robust to adjustments for other components of the metabolic syndrome (hypertriglyceridaemia, hypertension, hyperglycaemia and systemic inflammation). None of these components were themselves effect modifiers of the obesity-asthma association.

In this large, nationally representative sample, insulin resistance modified the association between obesity and current asthma in adults. Targeting insulin resistance may represent a novel therapeutic strategy for obese patients with asthma.

@ERSpublications

Insulin resistance potentiates the obesity-asthma association and may be a therapeutic target in obese asthmatics http://ow.ly/Zu9bb

Editorial comment in: Eur Respir J 2016; 48: 294-296.

This article has supplementary material available from erj.ersjournals.com

Received: Feb 012016 | Accepted after revision: March 102016 | First published online: April 212016

Support statement: This work was supported by grants from the National Institute of Allergy and Infectious Diseases, a National Institutes of Health Diversity Supplement (3U19AI095219-04S1), and funds from centre grant U19AI095219-01 to J.C. Cardet. Funding information for this article has been deposited with FundRef.

Conflict of interest: None declared.

Copyright OERS 2016 


\section{Introduction}

Obesity and asthma are common chronic diseases of public health importance [1-3]. Cluster analyses of asthma patients have revealed "obese asthma" as a relatively distinct subtype characterised by increased severity, resistance to usual therapy, late onset and female predominance [4-11]. Although the mechanisms underlying obese asthma are incompletely understood, systemic inflammation, adipokine alterations, and mechanical stress may each have a role in its pathobiology and blunted response to usual therapy [12-15]. Obesity as a clinical entity is heterogeneous [16] and may be associated with the metabolic syndrome, which is marked by dyslipidaemia, hypertension, hyperglycaemia and insulin resistance, central obesity, and a systemic inflammatory state [17].

As with the metabolic syndrome, asthma is also characterised by dysregulated inflammation. This has sparked interest in whether the metabolic syndrome constitutes a subtype of obesity that most associates with asthma. Several studies have suggested that each of the individual components of the metabolic syndrome are indeed associated with asthma, independently of obesity [18-20]. However, the relative contribution of each component of the metabolic syndrome to a more severe asthma phenotype has not been established, as recently reviewed by BAFF et al. [21]. Whether components of the metabolic syndrome are also effect modifiers of the association between obesity and asthma is unclear. Effect modification is distinct from confounding, and indicates that the magnitude of the association between obesity and asthma varies by the levels of a third variable, the effect modifier. That is, the effect modifier potentiates the association between obesity and asthma. To our knowledge, insulin resistance is the only component of the metabolic syndrome that has been reported to modify the effect of obesity on asthma, and this relationship has been shown only in children and adolescents [20], not in adults [22, 23].

Effect modification may not have been demonstrated in adults due to small sample sizes and because few study cohorts include data on insulin resistance. We hypothesised that insulin resistance is an effect modifier of the association between obesity and asthma in adults: that obese adults with the highest levels of insulin resistance are at greater odds of having asthma than obese adults with the lowest levels of insulin resistance. Using a large, nationally representative dataset, we show that obese adults have greater odds of current asthma with increasing levels of insulin resistance and not with increasing levels of other components of the metabolic syndrome.

\section{Methods}

Study design and population

Data were obtained from the 2003-2012 National Health and Nutrition Examination Survey (NHANES), a survey designed to sample the non-institutionalised US population. Participation in the survey is voluntary and informed consent was obtained from all participants. NHANES was approved by the Institutional Review Board of the National Center for Health Statistics of the US Centers for Disease Control and Prevention [24].

Data on sociodemographic factors, respiratory disease, medical conditions and household smoke exposure were obtained by questionnaire. Body measurements, spirometry and blood samples were acquired at a subsequent visit to a mobile NHANES clinical station. Height, weight and waist circumference were obtained on all subjects by trained health technicians using procedures described in the NHANES Anthropomorphic Procedures Manual [25]. Body mass index (BMI) was defined as weight in kilograms divided by the squared height in metres and was divided into traditional categories: $<25$ (lean), $\geqslant 25$ and $<30$ (overweight), and $\geqslant 30 \mathrm{~kg} \cdot \mathrm{m}^{-2}$ (obese). Obesity was also measured in terms of waist circumference and waist-to-height ratio.

Blood samples were processed to determine serum insulin, fasting total triglycerides, haemoglobin A1C $(\mathrm{HbA} 1 \mathrm{C})$ and C-reactive protein (CRP) levels in the morning after a 9-h fast. Spirometry was only available for survey years 2007-2012. Subjects were excluded from the spirometry portion of the exam if they reported current chest pain or a physical problem with forceful expiration; taking supplemental oxygen; recent surgery of the eye, chest or the abdomen; a recent heart attack or stroke; tuberculosis exposure; or recently coughing up blood. Adults with a personal history of detached retina or a collapsed lung and children with painful ear infections were also excluded. Spirometry was performed according to the American Thoracic Society guidelines using Ohio 822/827 dry-rolling spirometers (Ohio Medical, Gurnee, IL, USA). Forced expiratory volume $1 \mathrm{~s}$ (FEV1) \% predicted values were determined using NHANES III equations [26, 27].

A participant was considered to have "current asthma" if he or she responded affirmatively to the question, "Has a doctor or other health professional ever told you that you have asthma?", and to the question, "In the past 12 months have you had wheezing or whistling in your chest?". Race/ethnicity was self-reported and dichotomised into "Caucasian" and "non-Caucasian". The poverty income ratio (PIR), which is the ratio of family income to the poverty threshold, was used as a measure of socioeconomic status. Based on prior studies, insulin resistance was defined using the homeostatic model assessment of 
insulin resistance (HOMA-IR): insulin in milliunits per litre multiplied by fasting blood glucose in millimoles per litre divided by $22.5[20,28]$.

\section{Statistical analyses}

Statistical analyses were performed with STATA 12.0 software (StataCorp, College Station, TX, USA). Primary sampling units and strata variables provided in the NHANES dataset were used in the analysis to account for the complexity of the survey design. Sampling weights corresponding to each NHANES survey year were used to generate estimates representative of the US noninstitutionalised population.

The Chi-squared test was used to determine whether demographic characteristics were different between asthmatic and non-asthmatic adults for whom data on insulin resistance and covariates were available. This test was also used to compare demographic characteristics for those on whom data on insulin resistance were and were not available. Logistic regression was used to determine associations between the predictors "BMI categories" and "tertiles of insulin resistance", and the outcome "current asthma". All analyses were adjusted for age, sex, race/ethnicity, PIR and smoking status. Interaction terms were developed to test effect modification by insulin resistance (tertiles) on the association between current asthma and obesity (BMI, waist circumference, and waist-to-height ratio). The effect of insulin resistance on the association between obesity and current asthma was quantified by stratification by insulin resistance tertiles. Additional models were constructed with adjustments by other components of the metabolic syndrome (triglyceride levels, hypertension, HbA1C and CRP). Furthermore, interaction terms were developed to test effect modification by these components of the metabolic syndrome and obesity on the outcome of current asthma. Linear regression was used to compare lung function parameters (FEV1 \% predicted) between BMI categories, adjusting for age, sex, race/ethnicity, PIR, current asthma status and height. Again, the interaction term for "BMI $\times$ insulin resistance" was introduced to test whether significant spirometric differences exist between obese individuals with the highest versus lowest levels of insulin resistance.

\section{Results}

Data on current asthma and insulin resistance were available for 12421 participants from NHANES 2003-2012 (table 1). Of these, 6\% reported currently experiencing symptoms of asthma. As compared to adults without asthma, asthmatic adults were slightly younger, more likely to be female and less likely to be Caucasian; they had slightly lower PIRs and were more likely to be current smokers. Asthmatic adults were more likely to be obese (51 versus 33\%, $\mathrm{p}<0.001)$ and they had the highest levels of insulin resistance $(42 \%$ versus $32 \%, \mathrm{p}<0.001)$.

As expected, BMI was positively associated with current asthma (table 2). Relative to lean individuals, the odds of current asthma were 2.25 (95\% CI 1.63-3.10) times greater for obese participants, adjusting for

\begin{tabular}{|c|c|c|c|}
\hline \multirow[t]{2}{*}{ Characteristic } & \multicolumn{2}{|c|}{ Current asthma } & \multirow[t]{2}{*}{ p-value } \\
\hline & Yes & No & \\
\hline Subjects $\mathrm{n}$ & 752 & 11669 & \\
\hline Age years mean (so) & $47(29-65)$ & $49(30-67)$ & 0.01 \\
\hline Females & 60 & 51 & $<0.001$ \\
\hline Caucasians & 49 & 43 & 0.001 \\
\hline Insulin resistance & & & $<0.001$ \\
\hline Lowest tertile & 28 & 34 & \\
\hline Middle tertile & 30 & 34 & \\
\hline Highest tertile & 42 & 32 & \\
\hline Body mass index $\mathrm{kg} \cdot \mathrm{m}^{-2}$ & & & $<0.001$ \\
\hline$<25$ & 23 & 33 & \\
\hline$\geqslant 25-29.9$ & 25 & 34 & \\
\hline$\geqslant 30$ & 51 & 33 & \\
\hline Poverty index ratio $\geqslant 1$ & 79 & 89 & $<0.001$ \\
\hline Physician-diagnosed asthma & 100 & 8 & $<0.001$ \\
\hline Self-reported recent wheezing & 100 & 9 & $<0.001$ \\
\hline Smoking status & & & $<0.001$ \\
\hline Never & 42 & 54 & \\
\hline Former & 26 & 25 & \\
\hline Current & 32 & 21 & \\
\hline
\end{tabular}

Data are presented as \% unless otherwise stated. 
TABLE 2 Interaction between body weight and insulin resistance, and current asthma

Crude OR $(95 \%$ CI) Adjusted OR $(95 \% \mathrm{CI})$

Model 1: BMI categories
Lean
Overweight
Obese
Test for trend p-value
p-value for interaction between BMI and insulin resistance
Model 2: waist circumference tertiles
1st
2nd
3rd
Test for trend p-value
p-value for interaction between waist circumference
and insulin resistance
Model 3: waist-to-height ratio tertiles
1st
2nd
3rd
Test for trend ( $p$-value)
p-value for interaction between waist-to-height ratio
and insulin resistance

1.0 (ref.)

$1.01(0.73-1.38)$

$2.20(1.63-2.97)$

$<0.001$

0.10

1.0 (ref.)

$1.19(0.72-1.95)$

$2.17(1.35-3.47)$

$<0.001$

0.03

1.0 (ref.)

$0.97(0.65-1.44)$

$2.08(1.46-2.97)$

$<0.001$

0.11
1.0 (ref.)

$1.11(0.80-1.55)$

$2.25(1.63-3.10)$

$<0.001$

0.03

1.0 (ref.)

$1.38(0.84-2.29)$

$2.68(1.66-4.33)$

$<0.001$

0.01

1.0 (ref.)

$1.10(0.74-1.64)$

$2.29(1.56-3.35)$

$<0.001$

0.02

All models adjust for age, sex, race/ethnicity, poverty income ratio and smoking status. BMI: body mass index. Bold odds ratios are statistically significant.

age, sex, race/ethnicity, PIR, smoking status and insulin resistance level. Insulin resistance was not statistically significantly associated with current asthma $(\mathrm{p}=0.28)$ but the point estimate for current asthma was 7\% (95\% CI -5-21\%) greater for the highest tertiles of insulin resistance relative to the lowest tertile.

Having corroborated the association between BMI and asthma in our model, we then investigated our hypothesis of whether or not insulin resistance is an effect modifier of this association by introducing an interaction term. We found that insulin resistance is indeed an effect modifier of the relaptionship between obesity and asthma ( $p$-value for interaction 0.03) (table 2). Since obesity can be defined in ways other than BMI, such as waist circumference and waist-to-height ratio, we addressed the possibility that the significance of the interaction term between BMI and insulin resistance is due to assumptions inherent in the definition of "BMI". We therefore conducted similar analyses using interaction terms between insulin resistance and "waist circumference", and "waist-to-height ratio", respectively. The magnitude and significance of the effect estimates for the interaction terms for obesity defined as either waist circumference or waist-to-height ratio were similar to those for obesity defined as BMI (table 2).

Having identified insulin resistance as a statistically significant effect modifier, we then quantified the magnitude of this interaction by constructing a logistic regression model for current asthma and BMI, stratifying by tertiles of insulin resistance using the same prior adjustments. In this analysis, the odds ratio of current asthma for obese individuals relative to lean individuals increased with increasing tertiles of insulin resistance (OR 2.05, 2.76 and 3.00 for the three tertiles) (figure 1). This suggests that obese adults with the highest levels of insulin resistance have higher odds of being currently asthmatic than obese adults with the lowest levels of insulin resistance.

Insulin resistance is one of several other characteristics of the metabolic syndrome. We therefore considered the possibility that the significance of the above findings was due to confounding by one or more of the other components of the metabolic syndrome. To test this possibility, we first added each of the other components of the metabolic syndrome individually (serum triglyceride levels, hypertension, HbA1C and $\mathrm{CRP}$ ) to the original adjusted model in four additional models. None of these adjustments substantially changed the point estimates for the interaction term for BMI and insulin resistance, and the interaction term lost statistical significance only when adjusting by hypertension (p-value for interaction 0.06) (table 3). Second, we created interaction terms between BMI and each of the other components of the metabolic syndrome (triglyceride levels, hypertension, $\mathrm{HbA1C}$ and CRP) for the outcome of current asthma. We found that none of these interactions were statistically significant (table 4). This suggests that other components of the metabolic syndrome are neither confounders to the modifying effect of insulin resistance on the association between BMI and current asthma, nor effect modifiers themselves of that association. 
FIGURE 1 Relationship between between body mass index (BMI) and current asthma stratified by tertiles of insulin resistance. The $\mathrm{BMI}$ categories were lean $\left(<25 \mathrm{~kg} \cdot \mathrm{m}^{-2}\right)$ overweight $\left(\geqslant 25\right.$ and $\left.<30 \mathrm{~kg} \cdot \mathrm{m}^{-2}\right)$ and obese $\left(\geqslant 30 \mathrm{~kg} \cdot \mathrm{m}^{-2}\right)$. $\mathrm{p}$-value for interaction 0.03 .

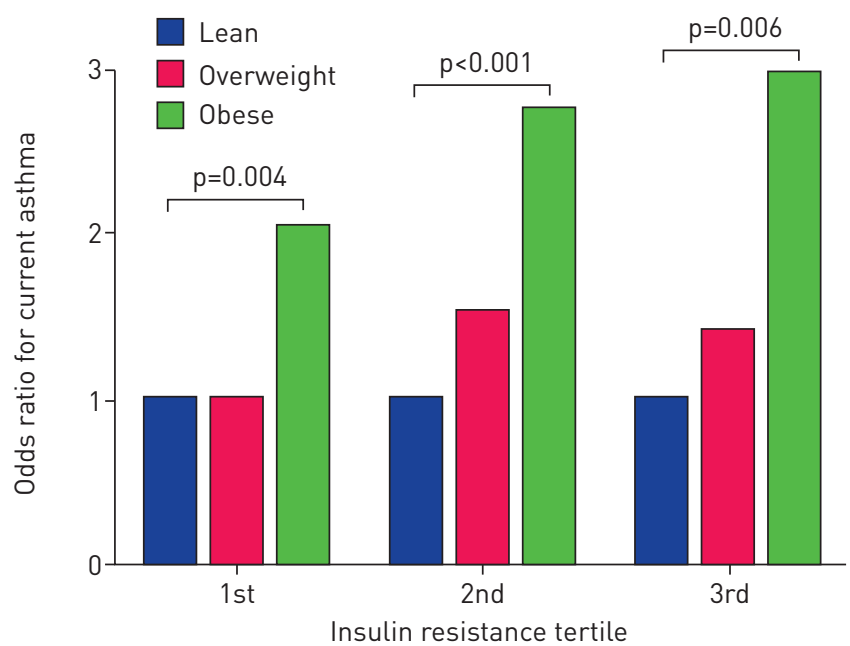

We then proceeded to investigate whether insulin resistance modifies the effect of obesity on objective metrics of airflow obstruction characteristic of asthma (spirometry). As expected, relative to lean individuals, obese individuals were found to have lower \% predicted FEV1 (1.18\% (95\% CI 0.1-2.27\%) lower, $\mathrm{p}=0.03$ ) adjusting for the same covariates as previously and for current asthma status. When we added insulin resistance to the model, we found that individuals with the highest levels of insulin resistance had lower \% predicted FEV1 than those with the lowest levels (4.71\% (95\% CI 3.27-6.15\%) lower, p<0.001). Having detected an association between both obesity and insulin resistance with the \% predicted FEV1, we then tested whether insulin resistance modifies the effect of obesity on spirometry. The interaction term for obesity and insulin resistance was statistically significant ( $p$-value for interaction 0.01 ). Relative to obese asthmatic adults with the lowest levels of insulin resistance, obese asthmatic adults with the highest levels of insulin resistance had a statistically significantly lower \% predicted FEV1 (1.15\% (95\% CI 0.35-1.95\%) lower). These analyses suggest that although insulin resistance modifies the effect of obesity on the prevalence of current asthma symptoms, the magnitude of its effect on airway obstruction is small.

Obese asthmatic adults with the highest levels of insulin resistance are clinically similar to those with lower levels of insulin resistance in terms of race/ethnicity, PIR and smoking status (online supplementary table S1). Although both groups were more likely to be female, obese asthmatic adults with the highest levels of insulin resistance were less represented by women than those with lower levels (57\% versus $78 \%$, $\mathrm{p}<0.001$ ). They were also slightly older (mean age 50 versus 47 years, $\mathrm{p}=0.03$ ).

\section{Discussion}

Using a large, nationally representative cohort, we have found that obesity is associated with asthma in adults, and that insulin resistance potentiates this association. Obese individuals with the highest levels of insulin resistance have greater odds of currently having asthma than obese individuals with the lowest levels of insulin resistance. Furthermore, we have found that effect modification is specific to insulin resistance and not to other components of the metabolic syndrome (hypertriglyceridaemia, hypertension, hyperglycaemia and systemic inflammation).

TABLE 3 The interaction between current asthma, body mass index (BMI) and insulin resistance is not substantially altered by adjustments with other components of the metabolic syndrome

Models with current asthma as outcome, with interaction term for "BMI $x$ insulin resistance"

Model 1: original model

Model 2: model 1 plus adjustment by triglyceride levels

Model 3: model 1 plus adjustment by hypertension

Model 4: model 1 plus adjustment by HbA1C levels

Model 5: model 1 plus adjustment by CRP levels p-value for

Point estimate for interaction term interaction term (95\% CI)

All models adjust for age, sex, race/ethnicity, poverty income ratio and smoking status. HbA1C: glycohaemoglobin; CRP: C-reactive protein. Bold point estimates are statistically significant. 
TABLE 4 Tests for interaction are negative between current asthma, obesity and components of the metabolic syndrome other than insulin resistance ${ }^{\#}$

BMI categories

Current asthma

Crude OR $(95 \% \mathrm{CI}) \quad$ Adjusted OR $(95 \% \mathrm{CI})$

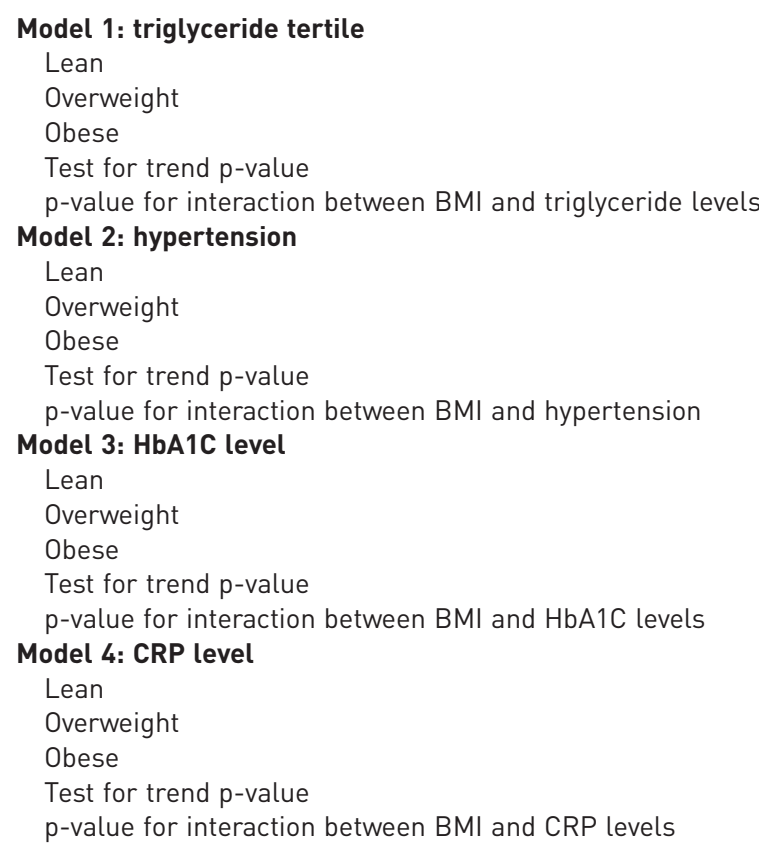

1.0 (ref.)

$1.12(0.89-1.42)$

$2.13(1.75-2.60)$

$<0.001$

0.54

1.0 (ref.)

$1.07(0.85-1.35)$

$1.94(1.58-2.38)$

$<0.001$

0.73

1.0 (ref.)

$1.12(0.89-1.41)$

$2.09(1.73-2.54)$

$<0.001$

0.19

1.0 (ref.)

$1.11(0.85-1.44)$

$1.96(1.59-2.41)$

$<0.001$

0.43

All models adjust for age, sex, race/ethnicity, poverty income ratio smoking status. BMI: body mass index; CRP: C-reactive protein; HbA1C: glycohaemoglobin. ${ }^{\#}$ : hypertriglyceridaemia, hypertension, diabetes and systemic inflammation (serum CRP levels). Bold odds ratios are statistically significant.

Our results are consistent with those of FonNo et al. [20], who documented insulin resistance as an effect modifier of obesity-asthma among children. Our findings expand on theirs in two ways: 1) by analysing the adult population and 2) by understanding effect modification by insulin resistance in the context of the other components of the metabolic syndrome. As a clinical entity, we expected extensive collinearity between other components of the metabolic syndrome and insulin resistance, which should bias results to the null. Conversely, our results on the modifying effect of insulin resistance were robust to adjustments by hypertriglyceridaemia, hypertension, hyperglycaemia and elevated levels of CRP. We did not detect effect modification of the obesity-asthma association with any of these other components of the metabolic syndrome. This suggests that insulin resistance, and not other components, is the feature of the metabolic syndrome that interacts with obesity to promote an asthmatic phenotype.

Although the mechanism by which insulin resistance potentiates the pro-asthmatic effects of obesity are unknown, we suspect that compensatory hyperinsulinaemia in the lung is responsible for the deleterious effects of insulin resistance. Insulin resistance occurs differentially in a signalling pathway- and cell type-specific manner [29-31]. The insulin receptor may become resistant to insulin agonism in some tissues (mainly the liver, adipocytes and skeletal muscle), more so than in the lung, which may leave pulmonary cells vulnerable to the effects of compensatory hyperinsulinaemia [32]. There are several pathways by which hyperinsulinaemia may impair pulmonary function, such as causing hypercontractility in airway smooth muscle cells $[29,30]$, reducing bioavailability of the endogenous bronchodilator nitric oxide [31], promoting mast cell survival [33] or fibroblast proliferation [34].

Our results suggest that the impact of insulin resistance as an effect modifier of the association between obesity and lung function is statistically significant but small (the \% predicted FEV1 of obese asthmatic adults with the highest levels of insulin resistance is only $1.15 \%$ lower than that of obese asthmatic adults with the lowest levels of insulin resistance). This may imply that the airway smooth muscle hypercontractility mechanism may predominate: insulin resistance may thus make asthmatic individuals more prone to be symptomatic with bronchoconstrictive stimuli yet not have more severe airflow limitation. Indeed, insulin therapy has been found to increase airway hyperresponsiveness in diabetic individuals [35]. The mechanism 
by which insulin resistance and hyperinsulinaemia potentiate the pulmonary effects of obesity needs to be investigated in in vitro and in vivo models.

This study has several limitations. First, data on insulin resistance were available for a subset of participants of NHANES 2003-2012. Missing data could result in overrepresentation of patient characteristics and bias our results. However, the subset for which insulin resistance data were available was not significantly different from the subset in which they was unavailable, in terms of sex, age, PIR, history of asthma, wheezing in the past year and smoking status (table S2). There were slightly more Caucasian participants in the subset on which insulin data was available (70\% versus 66\%) but, as previously shown, obese asthmatic adults with the highest insulin resistance levels were similar in their racial/ethnic composition as those with the lowest levels (table S1). This suggests that missing data probably do not contribute to our results. Second, spirometric data were only available on a subset of patients, but with this smaller subset, one would expect limitations in power and a bias towards the null, assuming that missingness is random. Despite this limitation, we did observe a statistically significant (albeit small) effect mediation by insulin resistance on the effect of obesity on pulmonary function. Third, we found that insulin resistance, and not other components of the metabolic syndrome, is an effect modifier of the obesity-asthma association. These negative results for other metabolic syndrome components could be due to limited power. However, the number of participants for which data on all other components of the metabolic syndrome was essentially the same as the number of participants for which we had data on insulin resistance. Although larger sample sizes could perhaps overcome this limitation, the effect size would also likely be small and of questionable clinical significance.

In summary, we have found, in a nationally representative cross-sectional study, that insulin resistance differentiates itself from other components of the metabolic syndrome in that it is the only component that potentiates the obesity-asthma association. This study suggests that insulin resistance distinguishes a distinct group of obese asthmatic adults. Therapies targeting insulin resistance to improve asthma control may benefit obese asthmatic adults with insulin resistance. Traditional insulin sensitisers [36], such as biguanides (like metformin) and thiazolidinediones (like pioglitazone), or emerging ones (such as free fatty acid receptor 1 agonists [37] or BGP-15 [38]), could be preferentially used in obese, insulin-resistant, asthmatic adults allowing a precision medicine approach. Additionally, it is possible that some of these drugs, metformin for example, which is now used for weight loss in nondiabetics with obesity, may have some benefit in obese asthmatics without diabetes. Both basic and prospective clinical research studies are needed to clarify whether organ-specific compensatory hyperinsulinaemia is the pathophysiological mechanism by which insulin resistance potentiates the association between obesity and asthma, and whether drugs that target insulin resistance can reduce asthma morbidity in obese patients.

\section{Acknowledgements}

The authors would like to thank E. John Orav and Ellen P. McCarthy from the Harvard School of Public Health (Boston, MA, USA) and Carrie Nettles from the BWH Asthma Research Center (Boston, MA, USA) for all their insights, help and support.

\section{References}

1 Ogden CL, Carroll MD, Kit BK, et al. Prevalence of childhood and adult obesity in the United States, 2011-2012. JAMA 2014; 311: 806-814.

2 Moorman JE, Zahran H, Truman BI, et al. Current asthma prevalence - United States, 2006-2008. MMWR Surveill Summ 2011; 60: Suppl., 84-86.

3 Moorman JE, Person CJ, Zahran HS. Asthma attacks among persons with current asthma - United States, 2001-2010. MMWR Surveill Summ 2013; 62: Suppl. 3, 93-98.

4 Gibeon D, Batuwita $\mathrm{K}$, Osmond $\mathrm{M}$, et al. Obesity-associated severe asthma represents a distinct clinical phenotype: analysis of the British Thoracic Society Difficult Asthma Registry Patient cohort according to BMI. Chest 2013; 143: 406-414.

5 Sutherland ER, Goleva E, King TS, et al. Cluster analysis of obesity and asthma phenotypes. PLoS One 2012; 7: e36631.

6 Telenga ED, Tideman SW, Kerstjens HA, et al. Obesity in asthma: more neutrophilic inflammation as a possible explanation for a reduced treatment response. Allergy 2012; 67: 1060-1068.

7 Sutherland ER, Goleva E, Strand M, et al. Body mass and glucocorticoid response in asthma. Am J Respir Crit Care Med 2008; 178: 682-687.

8 Rodrigo GJ, Plaza V. Body mass index and response to emergency department treatment in adults with severe asthma exacerbations: a prospective cohort study. Chest 2007; 132: 1513-1519.

9 Peters-Golden M, Swern A, Bird SS, et al. Influence of body mass index on the response to asthma controller agents. Eur Respir J 2006; 27: 495-503.

10 Camargo CA Jr, Boulet LP, Sutherland ER, et al. Body mass index and response to asthma therapy: fluticasone propionate/salmeterol versus montelukast. J Asthma 2010; 47: 76-82.

11 Boulet LP, Franssen E. Influence of obesity on response to fluticasone with or without salmeterol in moderate asthma. Respir Med 2007; 101: 2240-2247.

12 Sideleva O, Suratt BT, Black KE, et al. Obesity and asthma: an inflammatory disease of adipose tissue not the airway. Am J Respir Crit Care Med 2012; 186: 598-605. 
13 McLachlan CR, Poulton R, Car G, et al. Adiposity, asthma, and airway inflammation. J Allergy Clin Immunol 2007; 119: 634-639.

14 Al-Alwan A, Bates JH, Chapman D, et al. The non-allergic asthma of obesity: a matter of distal lung compliance. Am J Respir Crit Care Med 2014; 189: 1494-1502.

15 Moore WC, Meyers DA, Wenzel SE, et al. Identification of asthma phenotypes using cluster analysis in the Severe Asthma Research Program. Am J Respir Crit Care Med 2010; 181: 315-323.

16 Field AE, Camargo CA Jr, Ogino S. The merits of subtyping obesity: one size does not fit all. JAMA 2013; 310: 2147-2148.

17 Alberti KG, Eckel RH, Grundy SM, et al. Harmonizing the metabolic syndrome: a joint interim statement of the International Diabetes Federation Task Force on Epidemiology and Prevention; National Heart, Lung, and Blood Institute; American Heart Association; World Heart Federation; International Atherosclerosis Society; and International Association for the Study of Obesity. Circulation 2009; 120: 1640-1645.

18 Brumpton BM, Camargo CA Jr, Romundstad PR, et al. Metabolic syndrome and incidence of asthma in adults: the HUNT study. Eur Respir J 2013; 42: 1495-1502.

19 Al-Shawwa B, Al-Huniti N, Titus G, et al. Hypercholesterolemia is a potential risk factor for asthma. J Asthma 2006; 43: 231-233.

20 Forno E, Han YY, Muzumdar RH, et al. Insulin resistance, metabolic syndrome, and lung function in US adolescents with and without asthma. J Allergy Clin Immunol 2015; 136: 304-311.

21 Baffi CW, Wood L, Winnica D, et al. Metabolic syndrome and the lung. Chest 2016; 149: 1525-1534.

22 Ma J, Xiao L, Knowles SB. Obesity, insulin resistance and the prevalence of atopy and asthma in US adults. Allergy 2010; 65: 1455-1463.

23 Thuesen BH, Husemoen LL, Hersoug LG, et al. Insulin resistance as a predictor of incident asthma-like symptoms in adults. Clin Exp Allergy 2009; 39: 700-707.

24 National Health and Nutrition Examination Survey. About the National Health and Nutrition Examination Survey. www.cdc.gov/nchs/nhanes/about_nhanes.htm Date last accessed: July 21, 2015. Date last updated: February 3,2014 .

25 National Health and Nutrition Examination Survey. About the National Health and Nutrition Examination Survey. Questionnaires, Datasets, and Related Documentation. Date last accessed: July 21, 2015. Date last updated: October 30, 2015

26 Hankinson JL, Odencrantz JR, Fedan KB. Spirometric reference values from a sample of the general U.S. population. Am J Respir Crit Care Med 1999; 159: 179-187.

27 Miller MR, Hankinson J, Brusasco V, et al. Standardisation of spirometry. Eur Respir J 2005; 26: 319-338.

28 Wallace TM, Levy JC, Matthews DR. Use and abuse of HOMA modeling. Diabetes Care 2004; 27: 1487-1495.

29 Konner AC, Bruning JC. Selective insulin and leptin resistance in metabolic disorders. Cell Metab 2012; 16 : $144-152$.

30 Otero YF, Stafford JM, McGuinness OP. Pathway-selective insulin resistance and metabolic disease: the importance of nutrient flux. J Biol Chem 2014; 289: 20462-20469.

31 Agrawal A, Mabalirajan U, Ahmad T, et al. Emerging interface between metabolic syndrome and asthma. Am J Respir Cell Mol Biol 2011; 44: 270-275.

32 Biddinger SB, Kahn CR. From mice to men: insights into the insulin resistance syndromes. Annu Rev Physiol 2006; 68: 123-158.

33 Lessmann E, Grochowy G, Weingarten L, et al. Insulin and insulin-like growth factor-1 promote mast cell survival via activation of the phosphatidylinositol-3-kinase pathway. Exp Hematol 2006; 34: 1532-1541.

34 Dupont J, Khan J, Qu BH, et al. Insulin and IGF-1 induce different patterns of gene expression in mouse fibroblast NIH-3T3 cells: identification by cDNA microarray analysis. Endocrinology 2001; 142: 4969-4975.

35 Terzano C, Morano S, Ceccarelli D, et al. Effect of insulin on airway responsiveness in patients with type 2 diabetes mellitus: a cohort study. J Asthma 2009; 46: 703-707.

36 Derosa G, Maffioli P, Salvadeo SA, et al. Direct comparison among oral hypoglycemic agents and their association with insulin resistance evaluated by euglycemic hyperinsulinemic clamp: the 60's study. Metabolism 2009; 58: 1059-1066.

37 Darwish KM, Salama I, Mostafa S, et al. Design, synthesis, and biological evaluation of novel thiazolidinediones as PPAR $\gamma /$ FFAR1 dual agonists. Eur J Med Chem 2016; 109: 157-172.

38 Literati-Nagy B, Kulcsar E, Literati-Nagy Z, et al. Improvement of insulin sensitivity by a novel drug, BGP-15, in insulin-resistant patients: a proof of concept randomized double-blind clinical trial. Horm Metab Res 2009; 41: 374-380. 